Introduction: The distinction of medulloblastomas and ependymomas plays an important role in the care plans and prognosis of children. We aimed to investigate the role of magnetic resonance spectroscopy (MRS) in the differentiation between medulloblastomas and ependymomas in children. Materials and methods: The institutional review board approved this prospective study. The brain magnetic resonance imaging protocol including axial multivoxel spectroscopy with a TE of 144 ms was assessed in $49 \mathrm{pa}-$ tients, who were divided into 2 groups: 40 patients with medulloblastomas and 9 patients with ependymomas. Receiver operating characteristic (ROC) curve analysis and the Youden index were utilized to determine the best cut-off, sensitivity, specificity, and area under the curve (AUC) values of the independent spectroscopy parameters. Results: The choline level (Cho) and the choline/creatine (Cho/ Cr) and choline/N-acetyl aspartate (Cho/NAA) ratios of medulloblastomas were significantly higher than those of ependymomas $(p<0.05)$. A Cho/NAA cut-off value of 1.24 to predict the diagnosis of medulloblastoma yielded the highest AUC and sensitivity of $80.3 \%$ and $97.5 \%$, respectively, while a Cho cut-off value of 4.64 produced the highest specificity value of $88.9 \%$.

Conclusions: Our findings suggest that Cho and Cho/NAA derived from MRS could serve as differential factors between paediatric medulloblastomas and ependymomas. Among those, a Cho/NAA cut-off value of 1.24 to predict the diagnosis of medulloblastoma generated the highest accuracy.

Key words: medulloblastoma, ependymoma, magnetic resonance imaging, spectroscopy.

Contemp Oncol (Pozn) 2021; 25 (2): 95-99 DOI: https://doi.org/10.5114/wo.2021.105939

\section{The impact of magnetic resonance imaging spectroscopy parameters on differentiating between paediatric medulloblastoma and ependymoma}

\author{
Nguyen Minh Duc
}

Department of Radiology, Hanoi Medical University, Hanoi, Vietnam Department of Radiology, Pham Ngoc Thach University of Medicine, Ho Chi Minh City, Vietnam

Department of Radiology, Children's Hospital 2, Ho Chi Minh City, Vietnam

\section{Introduction}

Brain tumours can be divided into 2 categories: intra-axial and extra-axial. The position for intra-axial tumours can be either above or below the tentorium. Supratentorial tumours are more common than infratentorial tumours in adults, but not in children. The 3 most prominent infratentorial tumours in children are medulloblastoma, ependymoma, and pilocytic astrocytoma [1-6].

As a non-invasive and non-radiative diagnostic method, magnetic resonance imaging (MRI) is the best imaging modality for assessing brain tumours in children. Moreover, MRI can provide important information regarding correct diagnosis and treatment. Unfortunately, medulloblastoma and ependymoma-the two most common solid tumours located in posterior cranial fossa-might share similar imaging findings even though their treatments and prognoses are different. Therefore, it remains essential to distinguish between these 2 tumour types in clinical practice, especially prior to treatment planning [7-10].

Magnetic resonance spectroscopy (MRS) is an advanced method for investigating the active ingredients inside lesions and is widely used in the treatment of cancerous diseases. Prior knowledge of the concentration of active substances in a tumour can provide valuable information for differential diagnosis, treatment strategy, and prognosis. Several previous studies have used MRS to assess and distinguishing brain tumour types in both adults and children [8,10-13]. Therefore, in this clinical study, we investigated the impact of MRS parameters on differentiating between paediatric medulloblastoma and ependymoma.

\section{Material and methods \\ Patient population}

The institutional review board of Children's Hospital 2 approved this prospective study. Informed consent was provided from all patients' legal representatives before the MRI procedure. The study was conducted at Children's Hospital 2 over a period of 2 years, beginning in February 2019. A total of 49 patients were included: 40 children with medulloblastoma and 9 children with ependymoma. All patients included in this study underwent an MRI followed by surgery to obtain the histopathological results.

\section{Anaesthesia procedure}

The induction of anaesthesia was performed by intravenous injection of midazolam (5 mg/1 mL) at a dose of $0.1 \mathrm{mg} / \mathrm{kg}$ (Hameln Pharm GmbH, Ger- 
many), followed by propofol $1 \%$ anaesthetic $(10 \mathrm{mg} / 1 \mathrm{~mL})$ at a dose of 3 mg/kg (Fresofol, Fresenius Kabi GmbH, Austria).

\section{Magnetic resonance imaging procedure}

Paediatric patients were scanned with a 1.5-Tesla MRI machine (Philips, Best, The Netherlands). All paediatric patients were assessed by a multivoxel spectroscopy sequence with the following parameters: TR: 2000 ms; TE: $144 \mathrm{~ms}$; Flip angle: NA; Slice thickness: $15 \mathrm{~mm}$; Gap: NA; Field of view: $120 \times 120 \mathrm{~mm}^{2}$; Matrix: $10 \times 10 \mathrm{~mm}^{2}$; Plane: Axial; Number of Acquisitions: 1; Duration: 2.08 minutes. MRS parameters are automatically derived from spectroscopy sequences by utilizing the Spectroview software of Philips IntelliSpace Portal version 11.

\section{Spectroscopy parameters}

Spectroscopy parameters were comprised of $\mathrm{N}$-acetyl aspartate (NAA), choline (Cho), creatine ( $\mathrm{Cr}$ ), NAA/Cr, choline/creatine (Cho/Cr), and choline/N-acetyl aspartate (Cho/NAA) (Fig. 1, 2).

\section{Data analysis}

SPSS software version 26 (IBM Corp, Armonk, New York, USA) was used to perform the statistical analysis. Quantitative variables are presented as the median and interquartile range. Quantitative variables were compared using the $U$ Mann-Whitney test. Receiver operating char- acteristic (ROC) curve analysis and Youden index were performed to evaluate the cut-off point, accuracy, sensitivity, and specificity of the predictive model. The tests were considered statistically significant at $p<0.05$.

\section{Results}

The study comprised 49 paediatric patients with posterior cranial fossa tumours (median age $=7$ years; male/ female ratio $=32 / 17$ ), including 40 with medulloblastoma (median age $=7$ years; male/female ratio $=25 / 15$ ) and 9 with ependymoma (median age $=3$ years; male/female ratio $=7 / 2$ ).

The Cho, Cho/Cr, and Cho/NAA of medulloblastomas were significantly higher than these of ependymomas $(p<0.05$, Table 1$)$.

A Cho/NAA cut-off value of 1.24 to predict the diagnosis of medulloblastoma yielded the highest area under the curve (AUC) of $80.3 \%$ and the highest sensitivity of $97.5 \%$, while a Cho cut-off value of 4.64 produced the highest specificity value of $88.9 \%$ (Table 2, Fig. 3).

\section{Discussion}

In the current molecular era, recognizing the tumour's mitotic function and metabolism is crucial for recommending the appropriate therapies. Over the last few years, MRS as a non-invasive and non-ionizing diagnostic method has gained particular importance in the field of paediat-

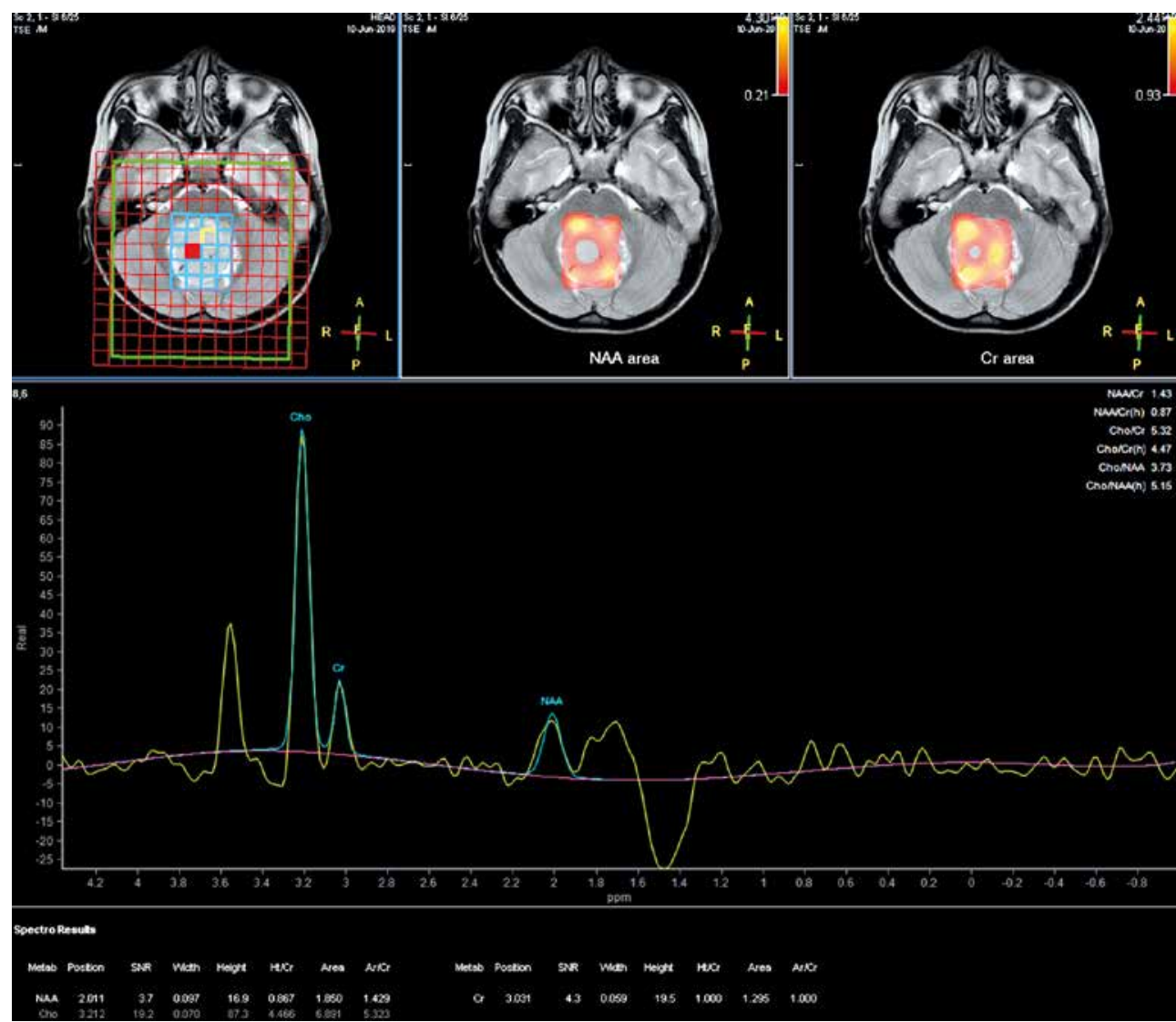

Fig. 1. An 8-year-old male patient presenting a tumour inside the fourth ventricle, confirmed as medulloblastoma after surgery. Magnetic resonance imaging spectroscopy indicates that the choline level was higher than the $\mathrm{N}$-acetyl aspartate level 


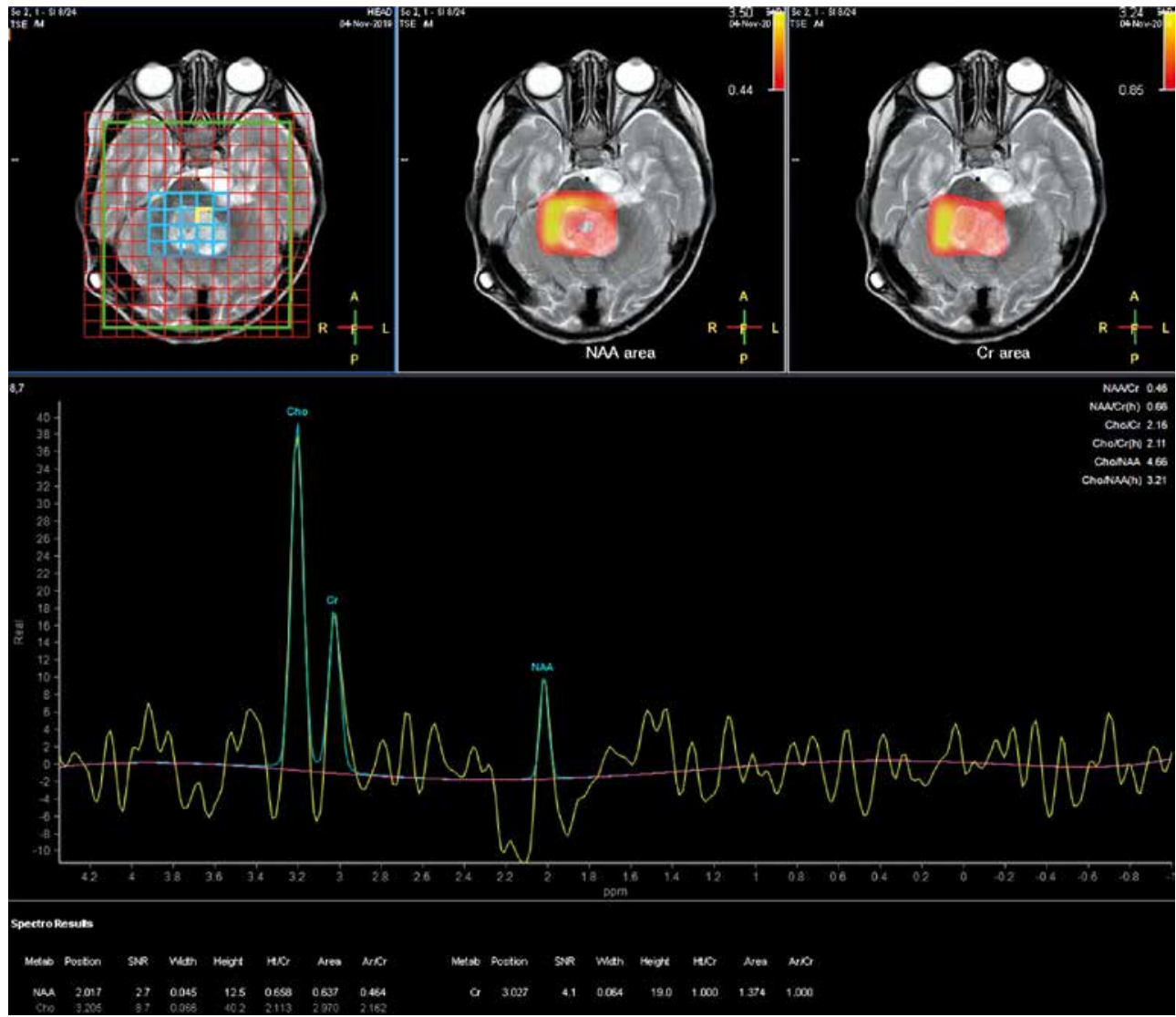

Fig. 2. A 3-year-old male patient had a tumour inside the fourth ventricle, confirmed as ependymoma after surgery. Magnetic resonance imaging spectroscopy shows that the choline level is higher than the $\mathrm{N}$-acetyl aspartate

ric brain tumours. MRS offers more information about the metabolic activity and cellular division of the tumours. Notably, Arle et al. used MRS to evaluate brain tumours in 33 children and observed different values of Cr/NAA, NAA/ $\mathrm{Cr}$, and $\mathrm{Cr} / \mathrm{Cho}$ ratios in PNET, meningeal, and astrocytomas [9]. In a previous study, it was observed that there was an increase in Cho and a decrease in NAA levels in PNET tumours [11]. Similarly, Chang et al. revealed that there was a decrease in NAA level with an increase in the Cho level in paediatric glioblastoma [10]. Moreover, Koob et al. used MRS in the classification and grading of paediatric brain tumours. MRS gave an accuracy of $49.7 \%$ in tumour classification and an accuracy of $65.9 \%$ in histological subdivisions [8].

Interestingly, Cuellar-Baena et al. also used MRS to study common types of posterior fossa brain tumours in children, including medulloblastoma $(n=8)$, ependymoma $(n=5)$, and pilocytic astrocytoma $(n=7)$. They noted a different spectrum of metabolite values among these 3 groups. The studied metabolites included fatty acids, Cho,
Table 1. Comparison of magnetic resonance spectroscopy parameters between medulloblastomas and ependymomas

$\begin{array}{lccc}\begin{array}{l}\text { Spectroscopy } \\ \text { parameters }\end{array} & \begin{array}{c}\text { Medulloblastoma } \\ n=40\end{array} & \begin{array}{c}\text { Ependymoma } \\ n=9\end{array} & p \\ \text { NAA } & 1.50(1.32) & 2.58(3.60) & 0.104 \\ \text { Cho } & 5.26(3.49) & 3.04(2.39) & 0.018 \S \\ \mathrm{Cr} & 1.29(1.09) & 1.00(1.21) & 0.929 \\ \mathrm{NAA} / \mathrm{Cr} & 1.41(1.7) & 2.68(4.4) & 0.313 \\ \mathrm{Cho} / \mathrm{Cr} & 4.28(4.42) & 2.16(3.08) & 0.020 \S \\ \text { Cho/NAA } & 3.54(4.14) & 1.22(2.43) & 0.004 \S\end{array}$

NNA - N-acetyl aspartate, Cho - choline, $\mathrm{Cr}$-creatin, $\mathrm{Cho} / \mathrm{Cr}$ - choline/creatine, Cho/NAA - choline/N-acetyl aspartate, $\S$-statistically significant

leucine, NAA, isoleucine, valine, $\gamma$-aminobutyric acid, acetate, glutamate, glutamine, glycine, myoinositol, creatine, serine, phenylalanine, and taurine [12]. In a previous study with MRS with a TE of 30 ms or 136 ms to distinguish between medulloblastoma, ependymoma, haemangioblastoma, and brain metastases, it was also revealed that

Table 2. Receiver operating characteristic analysis of magnetic resonance spectroscopy parameters for differential diagnosis between medulloblastomas and ependymomas

$\begin{array}{lccccc}\text { Spectroscopy parameters } & \text { Cut-off point } & \text { AUC } & \text { Sensitivity } & \text { Specificity } & 95 \% \mathrm{Cl} \text { interval } \\ \text { Cho } & 4.64 & 0.753 & 0.625 & 0.889 & 0.588-0.918 \\ \text { Cho/Cr } & 3.64 & 0.747 & 0.650 & 0.778 & 0.570-0.924 \\ \text { Cho/NAA } & 1.24 & 0.803 & 0.975 & 0.556 & 0.640-0.966\end{array}$

AUC - area under the curve, Cho - choline, Cho/Cr-choline/creatine, Cho/Naa - choline/N-acetyl aspartate, ${ }^{\S}$ - statistically significant 


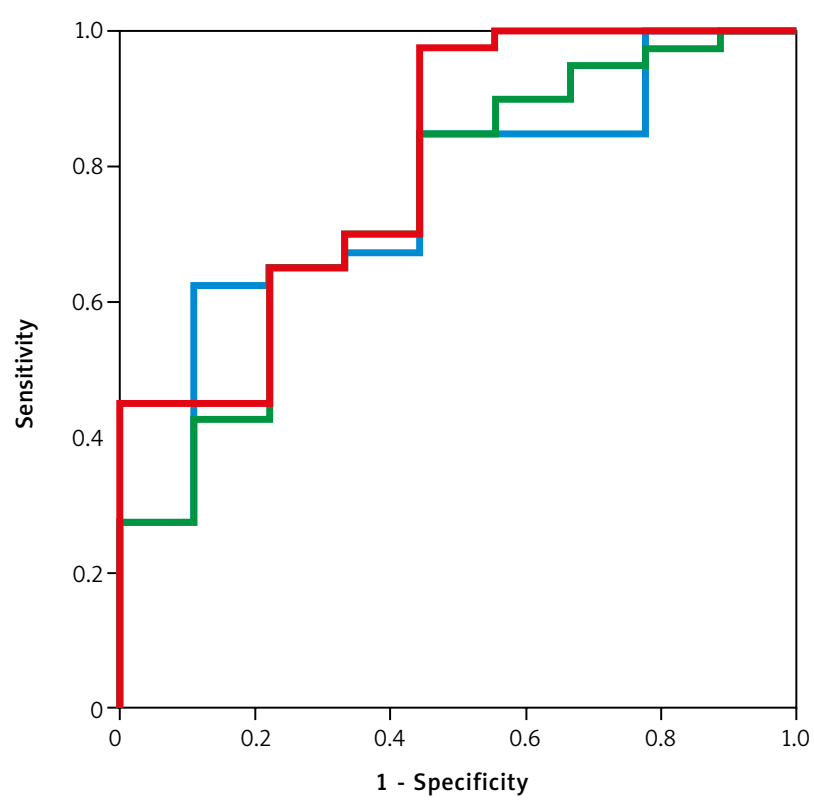

Choline Choline/Creatine Choline/N-acetyl/aspartate

Fig. 3. The receiver operating characteristic curves of choline, choline/creatine, and choline/N-acetyl aspartate

Cho/NAA ratio helped to distinguish between medulloblastoma and ependymoma with an accuracy of 92\% [13]. In this study, Cho and Cho/NAA levels were significantly lower in ependymomas compared to medulloblastoma. Thus, our findings are consistent with previous studies [8-13].

Understanding the genesis and function of both NAA and Cho could help in understanding the role it plays in tumour identification. Theoretically, NAA is synthesized in mitochondria from aspartate and acetyl-Co-A. It is then transported across the mitochondrial membrane and separated into aspartate and acetate by aspartoacylase in the cytoplasm. Therefore, it is suggested that NAA may function as a transporter of acetyl groups across the mitochondrial membrane to produce lipids during development. NAA is mostly concentrated in neurons and is considered a marker for normal neuron function. Brain tumours reduce the number of neurons/axons and reduce cellular density while impairing neuronal function, which often manifests as decreased NAA levels. Meanwhile, Cho concentration is closely related to cell membrane biochemistry, especially cell division. The concentration of Cho is considered a by-product of myelin breakdown. Therefore, when using spectroscopic MRI, cell growth associated with tumour growth may be accompanied by an increase in the Cho level [8-14].

Histopathologically, medulloblastoma is a malignant tumour that is typically characterized by very high cell density and high mitotic activity [15]. In contrast, ependymoma has a lower tumoral grade, lower cell density, and lower cell activity compared to medulloblastoma, which leads to a lower Cho and Cho/NAA ratio [8-14, 16]. Our findings are in line with these previous studies, and confirm that both Cho and Cho/NAA could serve as effective standards in distinguishing between 2 groups of medulloblastoma and ependymoma. We propose a Cho/NAA cut-off value of 1.24 to distinguish between these 2 diseases because the
AUC and sensitivity generated exhibited the highest value of $80.3 \%$ and $97.5 \%$, respectively. Meanwhile, a Cho cutoff value of 4.64 could be used because it has the highest specificity value of $88.9 \%$.

The small sample size and single-centre nature of the study could be regarded as a limitation of this research. Furthermore, the number of ependymoma patients is relatively low in this study. The researcher only intended to employ MRS to differentiate between paediatric medulloblastoma and ependymoma; therefore, the knowledge of morphological characteristics and conventional MRI features was insufficient. We used multivoxel MRS with TE $144 \mathrm{~ms}$ and focused on a few selected substances including NAA, Cho, and $\mathrm{Cr}$. We recommend that further studies with larger sample sizes and multicentre involvement should be performed to validate our findings. These studies should analyse the MRS with TE 35 ms and include a greater number of substances including myo-inositol, glutamate, and lactate. Additionally, future research could also combine both the conventional MRI and MRS to serve as a tool to distinguish between medulloblastoma and ependymoma.

\section{Conclusions}

Our findings suggest that Cho and Cho/NAA parameters could serve as differentiating factors between paediatric medulloblastomas and ependymomas. A Cho/NAA cut-off value of 1.24 could predict the diagnosis of medulloblastoma with the highest AUC of $80.3 \%$ and sensitivity of $97.5 \%$. Meanwhile, a Cho cut-off value of 4.64 produced the highest sensitivity value of $88.9 \%$. Nonetheless, further research should be conducted to validate these findings.

\section{The author declares no conflict of interest.}

\section{References}

1. Ostrom QT, Gittleman H, Xu J, et al. CBTRUS statistical report: primary brain and other central nervous system tumors diagnosed in the united states in 2009-2013. Neuro Oncol 2016; 18: v1-75.

2. Ostrom OT, de Blank PM, Kruchko C, et al. Alex's Lemonade stand Foundation Infant and Childhood Primary Brain and Central Nervous System Tumors Diagnosed in the United States in 2007-2011. Neuro Oncol 2015; 16: x1-36.

3. Duc NM, Huy HQ, Nadarajan C, Keserci B. The role of predictive model based on quantitative basic magnetic resonance imaging in differentiating medulloblastoma from ependymoma. Anticancer Res 2020; 40: 2975-2980.

4. Poretti A, Meoded A, Huisman TA. Neuroimaging of pediatric posterior fossa tumors including review of the literature. J Magn Reson Imaging 2012; 35: 32-47.

5. Tortori-Donati P, Fondelli MP, Cama A, Garre ML, Rossi A, Andreussi L. Ependymomas of the posterior cranial fossa: CT and MRI findings. Neuroradiol 1995; 37: 238-243.

6. Tortori-Donati P, Fondelli MP, Rossi A, et al. Medulloblastoma in children: CT and MRI findings. Neuroradiol 1996; 38: 352-359.

7. Duc NM, Huy HQ. Magnetic resonance imaging features of common posterior fossa brain tumors in children: a preliminary vietnamese study. Open Access Maced J Med Sci 2019; 7: 2413-2418.

8. Koob M, Girard N, Ghattas B, et al. The diagnostic accuracy of multiparametric MRI to determine pediatric brain tumor grades and types. J Neurooncol 2016; 127: 345-353.

9. Arle JE, Morriss C, Wang ZJ, Zimmerman RA, Phillips PG, Sutton LN. Prediction of posterior fossa tumor type in children by means of 
magnetic resonance image properties, spectroscopy, and neural networks. J Neurosurg 1997; 86: 755-761.

10. Chang YW, Yoon HK, Shin HJ, Roh HG, Cho JM. MR imaging of glioblastoma in children: usefulness of diffusion/perfusion-weighted MRI and MR spectroscopy. Pediatr Radiol 2003; 33: 836-842.

11. Chawla A, Emmanuel JV, Seow WT, Lou J, Teo HE, Lim CC. Paediatric PNET: pre-surgical MRI features. Clin Radiol 2007; 62: 43-52.

12. Cuellar-Baena S, Morales JM, Martinetto H, et al. Comparative metabolic profiling of paediatric ependymoma, medulloblastoma and pilocytic astrocytoma. Int J Mol Med 2010; 26: 941-948.

13. Mora P, Pons A, Cos M, et al. Magnetic resonance spectroscopy in posterior fossa tumours: the tumour spectroscopic signature may improve discrimination in adults among haemangioblastoma, ependymal tumours, medulloblastoma, and metastasis. Eur Radiol 2019; 29: 2792-2801.

14. Koob M, Girard N. Cerebral tumors: specific features in children. Diagn Interv Imaging 2014; 95: 965-983.

15. Koeller KK, Rushing EJ. From the archives of the AFIP: medulloblas toma: a comprehensive review with radiologic-pathologic correlation. Radiographics 2003; 23: 1613-1637.

16. Tamrazi B, Mankad K, Nelson M, D’Arco F. Current concepts and challenges in the radiologic assessment of brain tumors in children: part 2. Pediatr Radiol 2018; 48: 1844-1860.

\section{Address for correspondence}

\section{Dr. Nguyen Minh Duc}

Department of Radiology

Pham Ngoc Thach University of Medicine

Ho Chi Minh City, Vietnam

e-mail: bsnguyenminhduc@pnt.edu.vn

Submitted: 2.03.2021

Accepted: 26.03.2021 